\title{
Current scenario of dwarf threadworm, Strongyloides stercoralis infections in Southern Rajasthan, a systemic review
}

\author{
Bhumbla U. ${ }^{1 *}$, Kothari A. ${ }^{2}$, Rao J. ${ }^{3}$, Dalal A. ${ }^{4}$ \\ DOI: https://doi.org/10.17511/jopm.2020.i01.19 \\ 1* Upasana Bhumbla, Assistant Professor, Department of Microbiology, Geetanjali Medical College and Hospital, Udaipur, Rajasthan, India. \\ $\mathbf{2}$ Aditi Kothari, 2nd year Postgraduate, Department of Microbiology, Geetanjali Medical College and Hospital, Udaipur, Rajasthan, India. \\ 3 Jatin Rao, 2nd year Postgraduate, Department of Microbiology, Geetanjali Medical College and Hospital, Udaipur, Rajasthan, India. \\ ${ }^{4}$ A.S. Dalal, Professor and Head, Department of Microbiology, Geetanjali Medical College and Hospital, Udaipur, Rajasthan, India.
}

Strongyloides stercoralis is an intestinal nematode in humans. It is estimated that tens of millions people are infected worldwide, although no precise estimate is available. Although most infected individuals are asymptomatic, $S$. stercoralis is capable of transforming into a fulminant fatal illness under certain conditions associated with a compromise of host immunity. Strongyloides stercoralis has a complex biology with two separate life cycles, the freeliving cycle and the parasitic cycle. Filariform larvae in the soil infect the human host by penetration of intact skin to begin the parasitic cycle. The larvae enter circulation, are transported to the lungs, penetrate alveolar spaces, ascend the bronchial tree, are swallowed and reach the small bowel. The larvae migrate into the lumen and are either passed into feces or mature into filariform larvae, which can infect the intestinal mucosa or skin of the perianal region to restart the parasitic cycle. Rhabditiform larvae passed into feces can become infectious filariform larvae directly or go through a free-living cycle of development in the soil. This adaptability allows for the parasite's survival in the absence of mammalian hosts. Pulmonary symptoms such as a cough and tracheal irritation, mimicking bronchitis, occur as larvae migrate through the lungs several days later. Gastrointestinal symptoms (diarrhea, constipation, anorexia, abdominal pain) begin about 2 weeks after infection, with larvae detectable in the stool after 3 to 4 weeks. Techniques are periodically used to improve the sensitivity to detect Strongyloides larvae in stool samples. Various other standard methods that increase the sensitivity of diagnosis are larval concentration and culture methods, including Baermann, formalin-ethyl acetate sedimentation techniques, Harada-Mori, charcoal filter paper, and blood agar culture plate methods.

Keywords: Strongyloides stercoralis, Filariform, Rhabditiform

Corresponding Author

Upasana Bhumbla, Assistant Professor, Department of Microbiology, Geetanjali Medical College and Hospital, Udaipur, Rajasthan, India.

Email: ucupasana124@gmail.com
How to Cite this Article

To Browse

Bhumbla U, Kothari A, Rao J, Dalal AS. Current

scenario of dwarf threadworm, Strongyloides stercoralis infections in Southern Rajasthan, a systemic review. Trop J Pathol Microbiol. 2020;6(1):112-115 Available From https://pathology.medresearch.in/index.php/jopm/ar

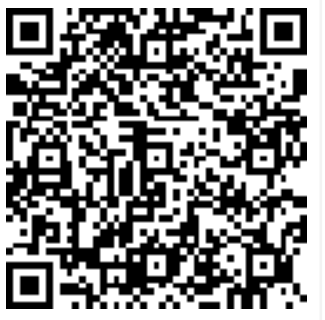
ticle/view/395

Manuscript Received 20-12-2019

Conflict of Interest No

Review Round 1
30-12-2019
Funding
Nil

Review Round 2
06-01-2020
Ethical Approval
Yes

Review Round 3

Accepted 10-01-2020

Plagiarism X-checker $16 \%$

(c) 2020 by Upasana Bhumbla, Aditi Kothari, Jatin Rao, A.S. Dalal and Published by Siddharth Health Research and Social Welfare Society. This is an Open Access article licensed under a Creative Commons Attribution 4.0 International License https://creativecommons.org/licenses/by/4.0/ unported [CC BY 4.0]. 


\section{Introduction}

In the last decade, interest in Strongyloides stercoralis infection and the disease complications has grown globally. The parasite is endemic in tropical and some temperate countries in the world where human fecal contamination of the soil is frequent; however, its prevalence has recently been estimated to be 370 million infections worldwide. Strongyloides is a parasite that is very prevalent in the tropical and subtropical regions of the world and is endemic in the South eastern United States and Asia including India. It has been estimated that there are 80-100 million cases worldwide. It is an important opportunistic pathogen in immunocompromised host. Strongyloidiasis is caused by the female nematode Strongyloides stercoralis [1]. In its classic life cycle, Strongyloides travels from the skin to the lungs and then to the gastrointestinal (GI) tract of its host. Strongyoidiasis, also known as dwarf thread worm which is caused by a smallest pathogenic nematode Strongyloides stercoralis, causes infection in man. It is unique as it has both parasitic and free-living generations. It is an ovoviviparous nematode. The filariform larvae are the infective stages of the parasite [2].

Infection usually results in asymptomatic chronic disease of the gut, which can remain undetected for decades. This can also lead to polymicrobial bacteremia, gram-negative bacillary meningitis, pneumonitis, and alveolar hemorrhage. However, hyperinfection syndrome and disseminated disease usually develop as a result of immune suppression caused by HIV infection, in addition to immunocompromised states like administration of corticosteroids, tumor necrosis factor-aantagonists, or transplantation. When immunosupression develops, autoinfection accelerates and hyperinfection occurs. Major complaints were fever, abdominal pain, diarrhea, abdominal distension, weight loss, vomiting, cough, anemia, and hemoptysis. It is associated with significant morbidity and mortality $[2,3]$.

\section{Life Cycle}

The S. stercoralis life cycle encompasses both freeliving and parasitic stages. Adult female worms parasitizing the human small intestine lay eggs in the intestinal mucosa that hatch into rhabditiform larvae, which are shed in the stool. In the environment, under warm moist conditions that
Often characterize the tropical and subtropical areas where S. stercoralis is endemic, rhabditiform larvae can either molt into infective filariform larvae or develop through succeeding rhabditiform stages into free-living adults. Harsh environmental conditions are thought to be a stimulus towards development into parasitic stages. Sexual reproduction occurs exclusively in the free-living stage.

Humans are generally infected by transcutaneous route, although infection has also been experimentally induced by oral administration of water contaminated with filariform larvae. After dermal penetration, the filariform larvae, through undefined mechanisms, migrate to the small intestine. The most clinically relevant, though perhaps not the predominant, migration is the classic pulmonary route, in which organisms enter the bloodstream and are carried to the lungs, ascending the tracheobronchial tree to enter the gastrointestinal tract.

Only female adults are detectable in humans, and subsequent reproduction occurs asexually. Some rhabditiform larvae transform into invasive filariform larvae before being excreted. As such, they are capable of reinfecting the host by invading the intestinal wall or the perianal skin. This auto infective cycle can occur at a low level throughout infection and allows subsequent generations to persist in the host indefinitely $[2,3,4]$.

The parasitological methods-based searches for larvae in the faeces are still the commonest choice of laboratory procedure for the diagnosis of this helminth. The stool sample is examined for saline mount which reveals the rhabditiform larva with a short buccal cavity. Three consecutive stool samples increases sensitivity to $70 \%-80 \%$. Formalin -ether or zinc flotation methods are also used to concentrate Strongyloides larvae in the stool. Stool culture is equally important in suspected cases of strongyloidiasis which are not confirmed either by direct smear or concentration methods and is done routinely. Stool is cultured either by Harada -Mori filter paper, Baermann funnel method using charcoal and agar plate method. Even screening for immune status is mandatory. Also, serological tests, skin tests and imaging techniques are used for the confirmation of diagnosis of Strongyoidiasis [5].

\section{Case Series}

Pulmonary strongyloidiasis: A 40-year female came to outpatient department with complaints of 
Fever for 3 months, cough with expectoration and decrease in appetite for 3 months. History of vomiting and headache for 2 days along with itching over the whole body was also present. On examination it was revealed, fever was high grade $1020 \mathrm{~F}$, not associated with chills and rigor, blood pressure was $120 / 80 \mathrm{mmHg}$. Cardivascular examination revealed no abnormal sounds, no murmurs; her chest examination had no unusual findings. Abdomen was soft with no organomegaly. Fundus examination revealed no papillodema, CT Head was normal. Laboratory evaluation results showed that patient was anemic (normocytic hypochromic), haemoglobin being $8 \mathrm{~g} / \mathrm{dl}$ andwhite blood cell count of 5100/cumm (N66E22L10M2). Urinalysis revealed grade $3+$ proteinuria, 5-10 white blood cells per high-power field, 3-4 red blood cells/hpf. Her LFT was relatively normal.Sputum smear for acid fast bacilli was negative whereas sputum for wet mount and stool examination revealed Strongyloides stercoralis larvae. Egg containing larva was also identified in the sputum sample. Patient was seropositive and her CD4 count was 39. She was already on anti-retroviral therapy [5].

Strongyloidiasis in HIV/AIDS: A 38-year-old female patient came to the outpatient department with complaints of intermittent fever, abdominal pain and frequent episodes of loose motions for 4 days. She had similar history of such complaints from past 5 months. On general physical examination pallor, pedal edema was noticed. Abdominal examination did not reveal distension, tenderness, organomegaly, lump or ascites. Blood picture showed haemoglobin $7 \mathrm{~g} / \mathrm{dl}$ (normal 12$15 \mathrm{~g} / \mathrm{dl}$ ) with microcytic hypochromic anemia, total leukocyte count of $4.7 /$ cumm; polymorphs $64 \%$, lymphocytes $32 \%$,eosinophil $2 \%$ and monocytes $2 \%$; ESR was $50 \mathrm{~mm} / \mathrm{hr}($ normal $<30)$. Her serum total protein and albumin were 60 and 30 respectively; creatinine was $53 \mu \mathrm{mol} / \mathrm{L}$ (normal 50 110), serum alanine and aspartate were 47 and 57U/L resp, alkaline phosphatase 170U/L. Her CD4 count was altered and patient was seropositive for HIV. HbsAg and HCV status were although negative. CT scan of abdomen and pelvis showed non-specific thickening of the intestinal wall. On X-ray patchy alveolar infiltrates were seen. In wet mount of stool, a Rhabditiform larva of Strongyloides stercoralis was identified by a short buccal cavity. Agar plate method done on blood agar plate, tracking of the larva was demonstrated, suggestive of Strongyoidiasis.
A $45 \mathrm{yr}$ old male patient came with complaints of abdominal pain and diarrhea for 1 month. His blood investigations revealed that patient was anemic (normocytic hypochromic), haemoglobin being 7.5 $\mathrm{g} / \mathrm{dl}$ andwhite blood cell count of 6200/cumm (N74E15L10M1). His liver function tests were normal but on stool examination it revealed Strongyloides stercoralis larvae. Further on Kinyon staining of stool sample, red colored oocysts of Isospora belli were also seen. Patient was seropositive and was further treated with Ivermectin and Cotrimoxazole and anti-retroviral therapy.

\section{Discussion}

The diagnosis of strongyloidiasis should be suspected in patients with clinical signs and symptoms of the disease (e.g. fever, cough, vomiting, and eosinophilia) or indicative serologic findings (e.g. increased IgE and positive enzymelinked immunosorbent assay results). Eosinophiliathe only clue to the infestation in many cases of Strongyoidiasis-might be suppressed or absent in disseminated disease because of a concomitant pyogenic infection or because of steroid administration. Serum IgE concentration is often elevated in patients with disseminated disease. Definitive diagnosis of strongyloidiasis is usually made by the detection of larvae in a stool sample.Many uncomplicated strongyloidiasis cases, however, have a very low intestinal worm load and minimal larvae excretion. The diagnostic sensitivity of larvae detection increases with repeated stool examinations $[2,3,6]$.

A study by Varatharajalu $R$, stated the different perspectives, wherein Strongyloidiasis is of primary medical importance, and fatal disease can occur in infected people who become immunosuppressed/immunocompromised through the administration of steroids or because of coinfection with human T-lymphotropic virus I [7]. In another study by Jourdan PM, the importance of Strongyloides stercoralis nematode and this parasite is rarely considered by soil-transmitted helminth control programmes, despite the harm potentially caused (hyperinfection being almost invariably fatal). It seems almost superfluous to say that the use of the most sensitive diagnostic techniques is mandatory to rule out the infection [8].

The diagnosis of strongyloidiasis depends on finding the larvae in routine fecal examinations, which are very insensitive in light infections when examining 
Only one specimen per patient. Repeated stool samples at different time intervals and the use of more sensitive methods of larval extraction such as Baermann and agar method yield better results, but are seldom used in public health facilities due to time, cost and demand of better trained laboratory personnel $[9,10]$. The introduction of screening tests such as ELISA or molecular techniques highly sensitive as a diagnostic tool for parasitic infections such as DNA detection using conventional or real time PCR promise better alternatives once certain difficulties are overcome such as cost and the requirement of better public health laboratory infrastructure in developing countries [11].

\section{Conclusion}

Definitive diagnosis of strongyloidiasis is generally made based on detection of larvae in stool, sputum, and duodenal fluid/biopsy. However, strongyloidiasis is difficult to diagnose only on stool examination as the parasite load is low and the larval output is irregular in the majority of patients. Although most studies focus on finding the parasite by means of stool examination, single stool examinations are unrewarding in up to $50-70 \%$ of cases. So in present study it was concluded that egg and the larval forms were detected in sputum as well as stool sample of patient. Along with Strongyloides stercoralis, oocysts of Isospora belli on Kinyon staining was equally pathogenic, wherein studies reveal that sometimes specimens are not able to recover oocysts due to its small number present in a given sample.

Ivermectin is the drug of choice for acute and chronic strongyloidiasis as it kills the parasite by binding selectively with glutamategated ion channels in muscles and nerve cells, resulting in cessation of cells. Malabsorption, haemorrhage and intestinal perforation are the commonest complications of strongyloidiasis. Important control measures should be taken which includes sanitary disposal of faeces, improved personal hygiene.

\section{Reference}

01. Blatt JM, Cantos GA. Evaluation of techniques for the diagnosis of Strongyloides stercoralis in human immunodeficiency virus (HIV) positive and HIV negative individuals in the city of Itajai, Brazil. Brazil J Infect Dis. 2003;7(6)402-408. doi:[Article:http://dx.doi.org/10.1590/S1413-86 702003000600008][Crossref]
02. Arora DR, Arora BB. Textbook of Medical Parasitology CBS Publishers. Isosporabelli. 3rd Edition 2013; Chapter 5;97-99.

[Crossref]

03. Gupta N, Choudhary A, Mirdha B R, Kale P, Kant K, Ghosh A. Strongyloides stercoralis InfectionA Case Series from a Tertiary Care Center in India. J Glob Infect Dis. 2017;9(2)86-87.

doi: [Article:https://doi.org/10.4103/0974777X.204694][Crossref]

04. Tripathi KD. Essentials of medical pharmacology. JP Medical Ltd. 6th edition; pp 764.

[Crossref]

05. Arakaki T, Iwanaga M, Kinjo F, Saito A, Asato R, Ikeshiro T. Efficacy of agar plate culture in detection of strongyloidesstercoralis infection. J Parasitol. 1990;76(3)425-428.

[Crossref]

06. Parija SC. Textbook of medical parasitology. 3rd edition. 2006, pp.

294-300 [Crossref]

07. Bhumbla U. Strongyloidiasis in seronegative patient- A case report. Med Sci. 2014;6(22)9496.

[Crossref]

08. Varatharajalu R, Kakuturu R. Strongyloides stercoralis- current perspectives. Dovepress. $2016 ; 5 ; 23-33$.

doi: [Article:https://doi.org/10.2147/RIP.S75839] [Crossref]

09. Jourdan PM, Lamberton PHL, Fenwick A, Addis DG. Soil-transmitted helminth infections. Lancet. 2017;391(10117)252-265.

doi: [Article:https://doi.org/10.1016/S01406736(17)31930-X][Crossref]

10. Hailemariam G, Kassu A, Abebe G, Abate E, Damte D, Mekonnen $E$, et al. Intestinal parasitic infections in HIV/AIDS and HIV seronegative individuals in a teaching hospital, Ethiopia. Jpn J Infect Dis. $2004 ; 57(2) 41-43$.

[Crossref]

11. Ghoshal UC, Alexender G, Ghoshal U, Tripathi S, Krishnani N. Strongyloides stercoralis infestation in a patient with severe ulcerative colitis. Indian J Med Sci. 2006;60(3)106-110.

doi: [Article:https://doi.org/10.4103/0019-

5359.22761][Crossref] 